\title{
bZIP transmembrane transcription factor CREBH: Potential role in non-alcoholic fatty liver disease (Review)
}

\author{
MIN WANG, SHUIPING ZHAO and MINGYUE TAN \\ Department of Cardiology, The Second Xiangya Hospital, Central South University, Changsha, Hunan 410000, P.R. China
}

Received March 27, 2015; Accepted December 2, 2015

DOI: $10.3892 / \mathrm{mmr} .2015 .4749$

\begin{abstract}
The cyclic adenosine monophosphate(cAMP)-responsive element-binding protein $\mathrm{H}(\mathrm{CREBH})$ is a transcription factor localized to the endoplasmic reticulum (ER) membrane. Previous studies have demonstrated that CREBH is activated by ER stress, hepatic glucose and lipid metabolism signaling, and inflammation. Thus, it may be critical in the regulation of various physiological functions associated with the development of non-alcoholic fatty liver disease (NAFLD), which results from inflammation and disorder of hepatic glucose and lipid metabolism. Therefore, CREBH may have potential as a pharmacological target for NAFLD. This review summarizes recent scientific developments and the biological actions of CREBH with a particular focus on its involvement in NAFLD.
\end{abstract}

\section{Contents}

1. Introduction

2. Regulation of CREBH expression

3. CREBH and hepatic lipid metabolism

4. CREBH and hepatic glucose metabolism

5. CREBH and inflammation

6. Roles of CREBH on NAFLD

7. Conclusions

\section{Introduction}

The endoplasmic reticulum (ER) is important in maintaining intracellular calcium stores, steroid and lipid biosynthesis, membrane regeneration, gluconeogenesis and the folding and assembly of newly synthesized proteins $(1,2)$. Accumulation

Correspondence to: Professor Shuiping Zhao, Department of Cardiology, The Second Xiangya Hospital, Central South University, 139 Renmin Road, Changsha, Hunan 410000, P.R. China

E-mail: zhaosp@medmail.com.cn

Key words: non-alcoholic fatty liver disease, cyclic adenosine monophosphate-responsive element-binding protein $\mathrm{H}$, glucose metabolism, lipid metabolism, inflammation of misfolded or unfolded proteins in the ER induces ER stress and results in the unfolded protein response (UPR). Activation of the UPR is primarily a protective mechanism for cells under stress, that results in the degradation of unfolded proteins accumulated in the ER by ER-associated degradation. The UPR system involves three major pathways dependent on inositol-requiring enzyme-1, activating transcription factor 6 (ATF6) and double-stranded RNA-dependent protein kinase-like endoplasmic reticulum kinase to accelerate unfolded protein degradation, increase the synthesis of chaperones and other proteins required for processing and suppress the synthesis of new proteins, respectively (3). UPR has been observed to be activated in numerous liver diseases, such as chronic viral hepatitis (4), insulin resistance (5), alcoholic liver disease $(6)$, ischemia-reperfusion injury $(7,8)$ and acute liver toxin insults (9). However, when these responses are activated to a high level and/or persistently, cells or organs undergo ER stress-induced injury (10-12).

The cyclic adenosine monophosphate (cAMP)-responsive element-binding protein $\mathrm{H}(\mathrm{CREBH})$, encoded by the CREB3L3 gene, is a type of ER-residing transcription factor that has a region of high sequence similarity with ATF6. It belongs to the CREB/ATF family (13), which also includes the following proteins: cAMP-responsive element-binding protein 3 (CREB3) also known as Luman or LZIP $(14,15)$; cAMP-responsive element binding protein 3-like 1 (CREB3L1) also known as OASIS (16); cAMP-responsive element binding protein 3-like 2 (CREB3L2) also known as $\mathrm{BBF} 2 \mathrm{H} 7$ (17); and cAMP-responsive element binding protein 3-like 4 (CREB3L4) also known as CREB4, AIBZIP or Tisp40 $(18,19)$. Although CREBH is robustly expressed in the liver, it is also expressed at lower levels in the small intestine and stomach $(13,20)$. CREBH contains an ER transmembrane domain, a transcription-activation domain and a basic leucine zipper (bZIP) domain. In response to ER stress, CREBH is activated by regulated intramembrane proteolysis and translocates from the ER to the Golgi apparatus, where it is cleaved by the site-specific proteases, site-1 protease and site- 2 protease, releasing bZIP (13). This forms the transcriptionally active form of CREBH, $\mathrm{CREBH}(\mathrm{N})$, which translocates to the nucleus $(21,22)$, similar to sterol regulatory element-binding transcription factor 1 (SREBP) and ATF6 $(23,24)$. Chan et al (25) observed that CREBH was modified at three $\mathrm{N}$-linked glycosylation sites in the luminal domain. Disruption of all three sites by site-directed mutagenesis abrogated 


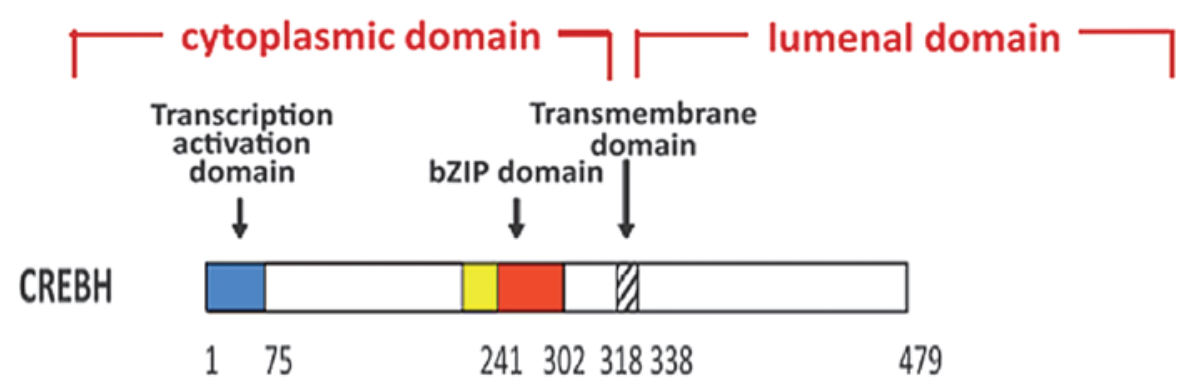

Figure 1. Protein structure of CREBH. CREBH has a transmembrane domain, a bZIP domain and a transcription activation domain. Adjacent to the N-terminal end of the bZIP region, 30 amino acids are conserved in CREBH and this is termed the ATB domain (indicated by the yellow box). CREBH, cyclic adenosine monophosphate-responsive element-binding protein $\mathrm{H}$; bZIP, basic leucine zipper.

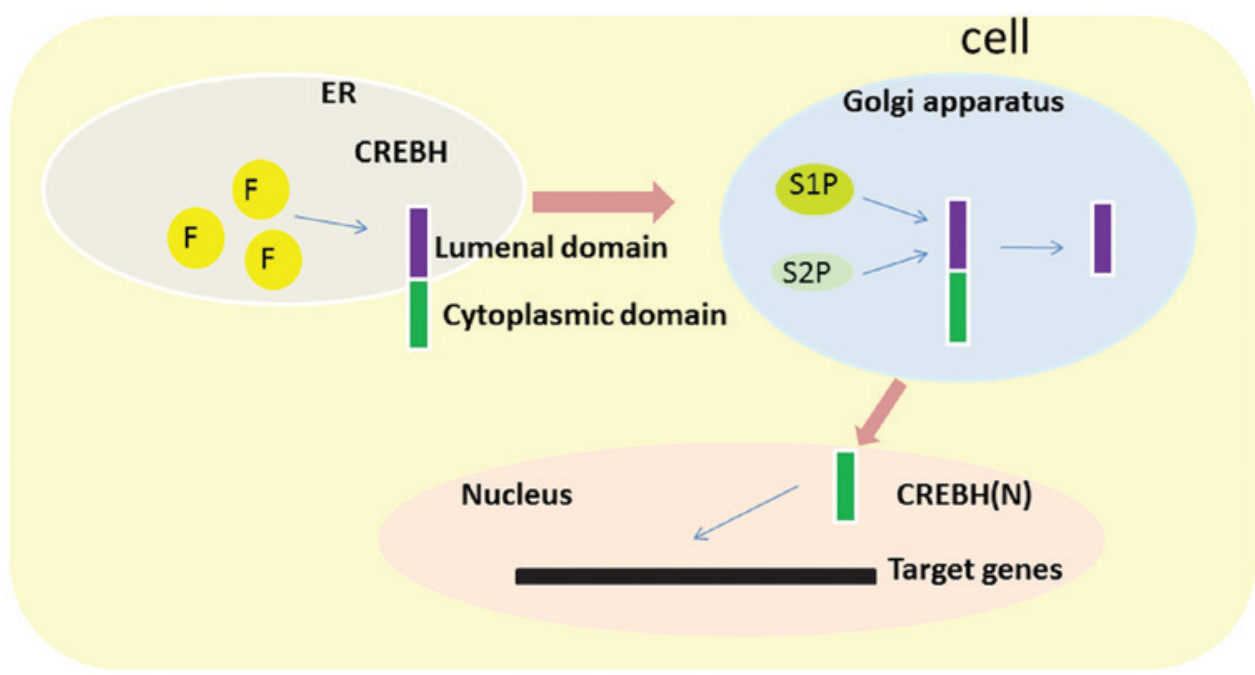

Figure 2. CREBH processing. Upstream factors activate CREBH in the ER. CREBH translocates to the Golgi apparatus and cleaved by S1P and S2P. The cleaved $\mathrm{N}$-terminal fragment of $\mathrm{CREBH}, \mathrm{CREBH}(\mathrm{N})$, translocates to the nucleus and regulates the expression of target genes. CREBH, cyclic adenosine monophosphate-responsive element-binding protein H; ER, endoplasmic reticulum; S1P, site-1 protease; S2P, site-2 protease; F, upstream factors.

$\mathrm{N}$-linked glycosylation of CREBH, and it was observed that $\mathrm{N}$-linked glycosylation is necessary for the activation of CREBH via intramembrane proteolysis (25). Unglycosylated CREBH is largely uncleaved and retained in an inactive form in the ER even if treated with an activator of ER stress and the UPR, such as brefeldin A, which induces proteolytic activation of CREBH (25). Unglycosylated CREBH is less capable of activating the transcription driven by the UPR element (25). Furthermore, Barbosa et al (26) reported that the CREB3 class of proteins has a highly conserved region designated the ATB domain, immediately adjacent to the $\mathrm{N}$-terminal end of the bZIP region and $\sim 30$ residues in length. It is observed only in the CREB3 class of proteins, however not in ATF6. The conserved ATB domain is required for CREBH in the context of physiological activation of target secretory pathway genes in human tissue culture cells and in Drosophila embryos (Figs. 1 and 2) (26).

Previous studies have demonstrated that following translocation to the nucleus, $\mathrm{CREBH}(\mathrm{N})$ binds to the CREBH-responsive element (CRE), box B and ATF6-binding element $(13,27,28)$ in the promoter of target genes involved in the regulation of the hepatic iron metabolism $(27,29)$, gluconeogenesis (30-32), lipid metabolism $(33,34)$ and inflammation $(22,35)$, and the pathophysiological procedure of
NAFLD (33) and associated conditions. The present review summarizes physiological functions of CREBH in NAFLD development and progression.

\section{Regulation of CREBH expression}

CREBH is activated by ER stress. An initial study demonstrated that CREBH expression was upregulated and the levels of $\mathrm{CREBH}(\mathrm{N})$ increased when cells were treated with ER stress inducers such as tunicamycin (TM), thapsigargin and dithiothreitol (22). However, subsequent studies did not observe the proteolytic activation of CREBH by ER stress inducer $(21,25,36)$. Xu et al (36) demonstrated that TM had no effect on CREBH mRNA expression levels and suppressed the processing of CREBH to $\mathrm{CREBH}(\mathrm{N})$ in vitro and in vivo, suggesting that CREBH processing is not increased by chemical ER stress-inducers. As mentioned above, the activation of CREBH via intramembrane proteolysis requires N-linked glycosylation; TM treatment inhibits CREBH glycosylation, thus, it is likely to result in the degradation of the unglycosylated CREBH protein (21). However, CREBH has been demonstrated to regulate the expression of hepcidin and proinflammatory and acute phase response genes, thus, linking ER stress to inflammation and iron metabolism $(20,27,37)$ and 
suggesting that the activation of CREBH in the context of ER stress is dependent on the specific agent and experimental conditions utilized. It remains to be determined whether CREBH processing is regulated under any physiologically relevant conditions in the liver.

CREBH is regulated by cannabinoid receptor type $1(C B 1 R)$ signaling. Previous studies investigating the underlying mechanisms of CB1R signaling in the regulation of hepatic gluconeogenesis demonstrated that $\mathrm{CREBH}$ is a downstream target gene of CB1R signaling, and is key in regulating hepatic gluconeogenesis (31), disrupting hepatic insulin receptor signaling (32) and mediating alcohol-induced regulation of bile acid enzyme gene expression (38). Following treatment with AM251 (a CB1R-specific antagonist), CB1R-mediated induction, by 2-AG (a CB1R agonist) of CREBH was reversed in rat hepatocytes (31). By pretreating primary hepatocytes with multiple specific inhibitors of cell signaling pathways prior to 2-AG treatment, Chanda et al (31) observed that CB1R signaling induces CREBH gene expression via the extracellular signal-regulated kinase $1 / 2$ and c-Jun N-terminal kinase (JNK) pathways. Furthermore, it was also demonstrated that an activator protein 1 binding site renders $2-\mathrm{AG}$ responsive to the CREBH promoter. Administration of 2-AG to cells with multiple serial deletion constructs of the $\mathrm{CREBH}$ promoter demonstrated a kinase-dead mutant of c-Jun (c-Jun KD; S63/73A) cotransfection significantly inhibited 2-AG-mediated activation of the CREBH gene promoter (31). Chanda et al (38) additionally observed that JNK phosphorylation and CREBH activation were significantly reduced in CB1R knockout mice challenged with alcohol. Overall, these data suggest that the $\mathrm{CB} 1 \mathrm{R}$ is a critical component for induction of $\mathrm{CREBH}$ via the JNK signaling transduction pathway.

CREBH is regulated by estrogen-related receptor- $\gamma$ (ERR $\gamma)$. The orphan nuclear receptor, ERR $\gamma$ is a constitutively active transcription factor that regulates genes involved in the hepatic glucose metabolism, alcohol metabolism and the ER stress response (39-41). Misra et al (35) reported that ERR $\gamma$ directly regulated CREBH gene expression in response to ER stress by binding to the ERR $\gamma$ response element in the CREBH promoter. Overexpression or knockdown of ERR $\gamma$ significantly increased or reduced, respectively, the expression of CREBH and C-reactive protein (CRP) (35). It also demonstrated the transcriptional coactivator, peroxisome proliferator-activated receptor $\gamma$ coactivator $1-\alpha(\mathrm{PGC} 1 \alpha)$ was required for $\mathrm{ERR} \gamma$-mediated induction of the $\mathrm{CREBH}$ gene by binding the CREBH promoter with ERR $\gamma$. The two proteins increased template-associated $\mathrm{H} 3$ and histone $\mathrm{H} 4$ acetylation to facilitate CREBH gene transcription (35). Previous studies have demonstrated that SHP-interacting leucine zipper protein (SMILE) inhibited the transactivation of ERR $\gamma$ by competition with transcriptional co-activator, $\mathrm{PGC} 1 \alpha(42,43)$. SMILE belongs to the bZIP family $(44,45)$, and the gene produces two isoforms, SMILE-L (long isoform, also termed CREBZF) and SMILE-S (short isoform, previously termed Zhangfei) (45). Misra et al (46) reported that curcumin and SMILE significantly inhibit the transcriptional activity of CREBH, however did not repress ATF6 transactivity. Following knockdown of endogenous SMILE, curcumin no longer inhibited the transcriptional activity of CREBH on the reporter gene, indicating that SMILE was a repressor of the transcription factor, CREBH45. They also demonstrated that SMILE interacted with CREBH via its bZIP domain, without being homodimerized, and competed with PGC1 $\alpha$ to inhibit CREBH transcriptional activity, similar to ERR $\gamma$ (46).

CREBH is regulated by nutrition. Hepatic CREBH is activated in a fasting state and markedly suppressed following refeeding (36). Long-term intake of a high-fat diet impairs the fasting/refeeding regulation (33) and these nutritional alterations in CREBH expression levels were markedly associated with plasma levels of free fatty acids (FFAs). Danno et al (47) demonstrated that CREBH expression increased in primary hepatocytes with the administration of various fatty acids (FAs). This is in agreement with the observations of Gentile et al (48), who also demonstrated that FAs upregulate CREBH via the activation of gene transcription. This processing is blocked by inhibitors of proteasome activity, suggesting that the upregulation of CREBH mRNA expression levels by FAs requires proteasome activity. Notably, insulin was indicated to prevent FFA-mediated upregulation of CREBH and the phosphatidylinositol-4,5-bisphosphate 3-kinase (PI3K) inhibitors affected the insulin-mediated suppression of the upregulation of CREBH47 by FFAs. This suggests that insulin and PI3K signaling are key determinants of the FFA-mediated regulation of CREBH in liver cells. The present review hypothesized that the suppression of CREBH mRNA expression levels in the fed state may be due to postprandial hyperinsulinemia. Glucocorticoids promote gluconeogenesis in an antagonistic effect to insulin by binding to the glucocorticoid receptor (GR) (49). Notably, dexamethasone, a synthetic corticosteroid, induces CREBH gene transcription by activating the binding of GR to the glucocorticoid transcriptional response element in the proximal promoter region (30). In addition, Danno et al (47) observed that the CREBH gene promoter contains a peroxisome proliferator responsive element for peroxisome proliferator-activated receptor (PPAR) $\alpha$ transactivation and indicated that administration of fenofibrate, a PPAR $\alpha$ agonist, increased CREBH expression. This induction was blocked by the further addition of a PPAR $\alpha$ inhibitor, MK886, suggesting that CREBH was regulated by PPAR $\alpha$. As FAs are endogenous ligands for PPAR $\alpha$, PPAR $\alpha$ is key in the adaptive response to fasting and increased concentrations of FAs, which may partially explain CREBH activation by fasting and FAs. However, another study (48) observed that the regulation of CREBH mRNA expression levels by FAs did not involve PPAR $\alpha$ signaling. Thus, it remains to be elucidated whether PPAR $\alpha$ is essential for FA- and fasting-induced upregulation of CREBH mRNA and protein expression levels. Toll-like receptor 4 (TLR4), similarly to PPAR $\alpha$, has emerged as an important mediator of the biological effects of FAs $(50,51)$. In H4IIE liver cells incubated with lipopolysaccharide (LPS), which activates TLR4, CREBH mRNA expression levels were observed to be significantly increased, suggesting that TLR4 signaling is also involved in the regulation of CREBH mRNA expression (48). Via TLR4-dependent pathways, gut microbiota, which increase serum LPS, have been suggested to be 
involved in the pathogenesis of NAFLD, due to the induction of liver inflammation (52).

CREBH is regulated by hepatocyte nuclear factor $4 \alpha$ $(H N F 4 \alpha)$. PPAR $\alpha$ has also been demonstrated to be regulated by HNF4 $\alpha$ (53), which is critical in hepatocyte differentiation and liver function (54). Luebke-Wheeler et al (20) demonstrated that HNF- $4 \alpha$ regulated CREBH directly via binding an $\mathrm{HNF} 4 \alpha$ recognition element site lying $3.7 \mathrm{~kb}$ upstream of CREBH exon 1, and that HNF4 $\alpha$ was important for the expression of CREBH in the liver, however not the small intestine (20). A possible explanation is that intestinal transcription factors, such as the HNF4 $\gamma$ protein that is predominantly expressed in the gut, regulate CREBH with no requirement for HNF4 $\alpha$. Further research to elucidate this disparity in expression is required.

\section{CREBH and hepatic lipid metabolism}

CREBH is a transcription factor that is key in the regulation of hepatic lipid accumulation. Following feeding with an atherogenic high-fat (AHF) diet, greater accumulation of hepatic lipid contents, such as hepatic triglycerides (TGs), plasma cholesterol, high-density lipoproteins, and low-density lipoproteins and an increase in plasma TG was observed in CREBH knockout mice than in control wild-type (WT) mice. Notably, the levels of plasma TG increased when feeding with a normal chow diet (33). To further investigate the involvement of CREBH in maintaining lipid homeostasis, CREBH null and WT control mice were fasted for $16 \mathrm{~h}$, and it was observed that the levels of plasma TG in the CREBH null mice were markedly increased compared with those of the control mice. Under nutrient starvation, a condition that stimulates lipolysis, the levels of ketone, 3-hydroxybutyric acid, a product of FA oxidation in the plasma, were slightly reduced in the CREBH-null mice, compared with the control mice, suggesting that CREBH deletion leads to a defect in TG lipolysis resulting in higher levels of plasma TG (33). Zhang et al (33) and Lee et al (34) demonstrated that plasma TG levels were significantly lower in $\mathrm{CREBH}(\mathrm{N})$ overexpression mediated by adenoviral infection or transgenic mice than in WT littermates. Zhang et al (33) demonstrated adenoviral delivery of activated CREBH resulted in a major increase in hepatic TGs.

CREBH regulates hepatic lipid accumulation predominantly by impacting the genes encoding the key enzymes involved in lipid metabolism. Gene microarray analysis and quantitative polymerase chain reaction analysis indicated that the deletion of CREBH in the liver reduced expression levels of five groups of genes involved in lipid metabolism, including lipogenic regulators, TG synthesis enzymes, enzymes or regulators in lipolysis and lipid transport, FA elongation enzymes and FA oxidation or cholesterol biosynthesis enzymes (33). Decreased expression levels of the lipogenic regulators and TG synthesis enzymes resulted in reduced de novo lipogenesis, and reduced expression levels of enzymes or regulators involved in FA elongation. Oxidation or cholesterol biosynthesis may be responsible for abnormal accumulation of hepatic lipid metabolites, and defective expression of enzymes required for lipolysis and lipid transport may account for hypertriglyceridemia, reduced fat mass and body weight gain, and massive steatosis in the CREBH null mice fed the AHF diet. The genes involved in regulation of CREBH in lipid metabolism in the liver include apolipoprotein (APO)C2, APOA4, APOA5, APOC3, fibroblast growth factor 21 (FGF21), fat-specific protein 27 (FSP27) and lipin 132 (34). APOC3 is a lipoprotein lipase (LPL) inhibitor, while APOA5 and APOC2 activate LPL. The activity of LPL is further increased by APOA4, facilitating the delivery of hydrolyzed FAs to peripheral cells, thus, lowering plasma levels of TG $(55,56)$. Patients with genetic defects in APOC2, APOA5 or LPL have high circulating TG levels due to impaired clearance (56-59). FGF21 is predominantly produced by the liver (60). Studies indicate that its concentration is correlated with hypertriglyceridemia, hepatic steatosis and insulin resistance (61-63). FSP27 is a lipid droplet (LD)-associated protein that promotes LD growth and TG storage in white adipocytes; it is also highly expressed in the steatotic liver and contributes to TG accumulation (64-66). Lipin 1 is a cytosolic phosphatidic acid phosphatase that generates diacylglycerol (DAG) in response to an increased intracellular FFA level, and is also crucial in lipid metabolism in the liver (67). CREBH regulates the transcriptional activation of APOC2, APOA4, APOA5, FGF21, FSP27 and lipin 1 directly by binding the CRE binding motifs in their gene promoters $(32,34,36,68-70)$. It remains unclear whether the APOC 3 promoter contains a CRE binding site, however, the very low density lipoprotein (VLDL)-associated APOC3 expression level is markedly higher in $\mathrm{CREBH}^{-} /$mice compared with WT mice, with no significant alterations in mRNA expression level (34), suggesting post-transcriptional control of APOC 3 by CREBH. Therefore, CREBH has a crucial role in the maintenance of hepatic TG homeostasis. Multiple nonsynonymous mutations in CREBH, including W46X, 245fs, E240K, V180M, G105R and P166L that produced nonfunctional or hypomorphic CREBH protein were identified in patients with extreme hypertriglyceridemia (34), further demonstrating a critical role for CREBH in the human TG metabolism. The identification of CREBH as a stress-induced metabolic regulator has important implications in the understanding and treatment of metabolic diseases.

\section{CREBH and hepatic glucose metabolism}

Phosphoenolpyruvate carboxykinase (PEPCK-C) and glucose-6-phosphatase (G6Pase) are important in the regulation of gluconeogenesis $(71,72)$. $\mathrm{CREBH}(\mathrm{N})$ has been demonstrated to increase PEPCK-C and G6Pase transcription by binding CRE in the promoters of these genes via a CREB/CREB-regulated transcriptional coactivator 2 (CRTC2)-dependent manner (30). Notably, knockdown of hepatic CREBH by small hairpin RNA results in reduced hepatic glucose production by suppressing PEPCK-C and G6Pase expression (30). Chanda et al (31) observed that CB1R signaling mediated by CREBH induced hepatic gluconeogenesis in primary hepatocytes; and the knockdown of CREBH attenuated the CB1R signaling-mediated upregulation of hepatic gluconeogenesis.

In addition, CREBH serves a potential role in the regulation of insulin resistance (INR). Notably, iron excess has been 
associated with reduced insulin sensitivity and with disease progression, whereas iron removal has been demonstrated to be beneficial (73-75). Iron metabolism is controlled by hepcidin, which is a downstream target gene of CREBH (27). Thus, CREBH has a critical association with insulin sensitivity. Hepatic CB1R and CREBH gene expression levels are higher in various models of insulin resistance $(30,76)$. Chanda et al (32) demonstrated that CB1R-mediated activation of $\mathrm{CREBH}$ increased DAG production and phosphorylation of protein kinase $\mathrm{C} \varepsilon$ type, which induces INR effects via disrupting the insulin receptor signaling pathway. CB1R activation under CREBH-deficient conditions failed to induce DAG production, ultimately leading to the recovery of insulin receptor signaling component activity. Therefore, the suppression of CB1R/CREBH signaling may reduce INR. However, the study by Chanda et al (32) did not directly assess insulin resistance in mice, such as with an insulin tolerance test. As mentioned above, fenofibrate, a PPAR $\alpha$ agonist, has been demonstrated to reduce plasma glucose and insulin levels and to increase insulin sensitivity mediated by CREBH (77). By contrast, Zhang et al (33) observed that CREBH knockout mice are less responsive to insulin than WT mice, which does not support the hypothesis that targeted inhibition of CREBH would be useful for improving insulin resistance. Therefore, the function of CREBH in controlling insulin sensitivity remains to be determined, however, these findings in rodent and human primary hepatocytes indicate that $\mathrm{CREBH}$ is important in the hepatic glucose metabolism.

\section{CREBH and inflammation}

CREBH is crucial in the activation of an acute inflammatory response. The acute phase response (APR) is systemic inflammatory component of innate immunity, which is an ancient metazoan adaptation mechanism initiated by chemical structures presented by invading microorganisms or exposed by damage to the host (78-80). Zhang et al (22) demonstrated that the expression levels of C-reactive protein (CRP) and serum amyloid P-component, whose synthesis occurs predominantly in APR, were significantly reduced in the fetal livers of CREBH knockdown mice compared with the RNAi control mice. It was also observed that CREBH and ATF6 interact and bind to the same conserved element in the promoter of CRP to synergistically activate expression upon ER stress, suggesting that CREBH was required to activate the APR. In addition, in line with other studies by Zhang et al (22) and Misra et al (35) it was demonstrated that ERR $\gamma$ increased CRP promoter activation via regulating $\mathrm{CREBH}$. $\mathrm{CREBH}$ expression was also observed to be induced by ER stress and proinflammatory cytokines, including interleukin (IL)6, IL1 $\beta$ or tumor necrosis factor $\alpha(\mathrm{TNF} \alpha)$, in hepatoma cells and in the liver. Notably, in ER stress, cleavage of CREBH, and APR induced by proinflammatory cytokines, occurred in the liver, but not in hepatoma cell lines; suggesting this is impaired and not comparable to that in the liver in vivo (22). Furthermore, hepcidin, another APR protein, is important in the anemia of inflammation (81) and is also transcriptionally regulated by CREBH (27,29). In addition, mRNA expression levels of other major APR proteins, including serum amyloid A1, A2 and A3, fibrinogen and $\alpha 1$-acid glycoprotein in CREBH knockdown fetal livers, were similar to those in the control fetal livers, suggesting that $\mathrm{CREBH}$ is not required for induction of all APR genes (22).

\section{Roles of CREBH on NAFLD}

NAFLD is defined as a pathological accumulation of fat in the form of TG in the liver, not as a result of alcohol consumption (82). It is an inclusive term that includes a spectrum of liver pathologies from simple steatosis to non-alcoholic steatohepatitis (NASH). The liver is important in the lipid metabolism, including importing and manufacturing FFAs, storing TGs in LDs and exporting lipids as VLDL to the serum. Alterations in any of these processes may result in the development of NAFLD (83). Donnelly et al (84) observed that under pathophysiological conditions, $\sim 60 \%$ of hepatic TGs derive from FFAs from adipose tissues, 26\% from de novo lipogenesis and $15 \%$ from the diet. FFAs derived from adipose tissues and de novo lipogenesis are termed non-esterified fatty acids. The excess of FFAs stimulates TG synthesis. The TGs are stored as LDs within hepatocytes, or lipidated by apolipoprotein B100 within the lumen of ER and subsequently secreted into the blood as VLDLs (85) via the Golgi apparatus (86). Therefore, an excess of FFAs and TG, or impaired VLDL assembly or secretion, may result in excessive lipid accumulation in the liver. Accumulation of lipids in the liver further stimulates existing hepatic INR by generation of lipid-derived secondary messengers, such as DAG and ceramides (87). Furthermore, lipid accumulation in the liver is also linked with the progression of ER stress, mitochondria stress and impaired autophagy, resulting in the condition termed lipotoxicity (88). This latter event may result in an immune response by the Kupffer cells and hepatic stellate cells, which leads to the progression of NASH, hepatic cirrhosis, and in certain severe cases, hepatocellular carcinoma (89).

In vivo data indicates that in metabolic syndrome disorders, such as NAFLD, levels of ER stress markers in the liver and other tissues are increased, and liver damage occurs (80-93). CREBH is an ER-bound transcription factor activated by ER stress and it is a key metabolic regulator required to activate expression of the genes involved in de novo lipogenesis, TG and cholesterol biosynthesis, FA elongation and oxidation, lipolysis and lipid transport in response to ER stress (33). Previous studies have demonstrated that fasting- and high fat diet (HFD)-induced fatty liver was more pronounced in $\mathrm{CREBH}^{-}{ }^{-}$mice compared with the WT mice $(33,70)$, while adenoviral delivery of CREBH inhibited HFD-induced steatosis in WT mice (77). In addition, NAFLD is associated with inflammation and fibrosis, increased hepatocyte ballooning, lobular and portal inflammation, Mallory bodies and collagen deposition were observed in CREBH null mice following the AHF diet (33). A histological scoring system for NAFLD was used $(94,95)$, demonstrating that the CREBH null mice developed profound NASH following the AHF diet. Increased levels of the key indicators of hepatotoxicity were also observed, including alanine aminotransferase and aspartate aminotransferase, and NASH-associated pro-inflammatory cytokines (96), such as TNFa and IL6 (33). Notably, fasting induced hepatic steatosis and an increase in 
CREBH expression levels (36), however, increased expression levels of CREBH maintains lipid homeostasis by regulating expression of the genes involved in the lipid metabolism (33). CREBH also inhibited HFD-induced steatosis in WT mice (77), suggesting there is a negative feedback control mechanism in the involvement of CREBH in the development of NAFLD.

CREBH is suggested to be involved in the development of NAFLD by regulating the lipid metabolism and maintaining insulin sensitivity. Accumulation of lipids in the liver stimulates existing hepatic insulin resistance, which further stimulates hepatic SREBP-1c production, resulting in increased de novo synthesis of fatty acids (97). As mentioned above, CREBH regulates insulin sensitivity by regulating hepcidin to control iron levels and mediating CB1R to disrupt hepatic insulin receptor signaling. Fenofibrate has been demonstrated to reduce plasma glucose and insulin levels and increase the insulin sensitivity mediated by CREBH (77). In addition, CREBH prevents insulin-induced SREBP-1c expression by markedly increasing the promoter activity of insulin induced gene 2, whose downregulation mediates insulin-stimulated transcriptional activity of SREBP-1c (77). The previous studies demonstrated that CREBH is key in the involvement of NAFLD by regulating insulin sensitivity.

As mentioned above, CREBH is also associated with inflammation in the liver $(22,35)$. The development of NAFLD involves insulin resistance and increased inflammation. Furthermore, inflammation in the development of NASH further impedes insulin signaling (98). Cytokine production of IL- 6 and TNF- $\alpha$ is increased in NASH and may be involved in its pathogenesis (99). CREBH expression and cleavage may be induced by IL- 6 and TNF- $\alpha$ to activate the APR (22), thus, the present review hypothesized that CREBH is a protective factor in response to inflammation by maintaining the balance of glucose and lipid metabolism by mediating gene expression in the liver for the two processes. In addition, TLR4 is activated by LPS or gut microbiota, which is also associated with NAFLD. The activated TLR4 increases hepatic expression levels of TNF- $\alpha$ and increases hepatic steatosis and inflammation during the development of NAFLD (52). CREBH is regulated by TLR4 (48), suggesting that CREBH may be activated by TLR4 and is, thus, involved in the development of NAFLD. The biological relevance of this regulation remains to be elucidated but CREBH may provide a potential therapeutic target in NAFLD.

\section{Conclusions}

Various studies have elucidated the roles of CREBH. Via the regulation by ER stress, CB1R signaling, ERR $\gamma$, SMILE, nutrition, PPAR $\alpha$ and HNF4 $\alpha$, CREBH promotes the genes encoding lipogenic regulators, TG synthesis enzymes, enzymes or regulators in lipolysis and lipid transport, FA elongation enzymes and FA oxidation or cholesterol biosynthesis enzymes to regulate hepatic lipid metabolism. By binding CRE in the promoter of PEPCK-C and G6Pase, it is involved in hepatic glucose metabolism in a CRTC2-dependent manner. In addition, CREBH regulates iron metabolism and mediates CB1R signaling, thereby regulating insulin sensitivity. Furthermore, CREBH is crucial in the activation of an acute inflammatory response. As inflammation and disorders of glucose and lipid metabolism are the predominant factors in NAFLD, targeting CREBH appears to be a promising effective therapeutic strategy.

\section{References}

1. Fujimoto M and Hayashi T: New insights into the role of mitochondria-associated endoplasmic reticulum membrane. Int Rev Cell Mol Biol 292: 73-117, 2011.

2. Wagner M and Moore DD: Endoplasmic reticulum stress and glucose homeostasis. Curr Opin Clin Nutr Metab Care 14: 367-373, 2011.

3. Esposito V, Grosjean F, Tan J, Huang L, Zhu L, Chen J, Xiong H, Striker GE and Zheng F: CHOP deficiency results in elevated lipopolysaccharide-induced inflammation and kidney injury. Am J Physiol Renal Physiol 304: F440-F450, 2013.

4. Benali-Furet NL, Chami M, Houel L, De Giorgi F, Vernejoul F, Lagorce D, Buscail L, Bartenschlager R, Ichas F, Rizzuto R and Paterlini-Bréchot P: Hepatitis C virus core triggers apoptosis in liver cells by inducing ER stress and ER calcium depletion. Oncogene 24: 4921-4933, 2005.

5. Ozcan U, Cao Y, Yilmaz E, Lee AH, Iwakoshi NN, Ozdelen E, Tuncman G, Görgün C, Glimcher LH and Hotamisligil GS: Endoplasmic reticulum stress links obesity, insulin action, and type 2 diabetes. Science 306: 457-461, 2004.

6. Ji C and Kaplowitz NL: Betaine decreases hyperhomocysteinemia, endoplasmic reticulum stress, and liver injury in alcohol-fed mice. Gastroenterology 124: 1488-1499, 2003.

7. Duvigneau JC, Kozlov AV, Zifko C, Postl A, Hartl RT, Miller I, Gille L, Staniek K, Moldzio R, Gregor W, et al: Reperfusion does not induce oxidative stress but sustained endoplasmic reticulum stress in livers of rats subjected to traumatic-hemorrhagic shock. Shock 33: 289-298, 2010.

8. Emadali A, Nguyên DT, Rochon C, Tzimas GN, Metrakos PP and Chevet E: Distinct endoplasmic reticulum stress responses are triggered during human liver transplantation. J Pathol 207: $111-118,2005$.

9. Kim TH, Kim YW, Shin SM, Kim CW, Yu IJ and Kim SG: Synergistic hepatotoxicity of N,N-dimethylformamide with carbon tetrachloride in association with endoplasmic reticulum stress. Chem Biol Interact 184: 492-501, 2010.

10. Ron D: Translational control in the endoplasmic reticulum stress response. J Clin Invest 110: 1383-1388, 2002.

11. Kaufman RJ: Orchestrating the unfolded protein response in health and disease. J Clin Invest 110: 1389-1398, 2002.

12. Schröder M and Kaufman RJ: ER stress and the unfolded protein response. Mutat Res 569: 29-63, 2005.

13. Omori Y, Imai J, Watanabe M, Komatsu T, Suzuki Y, Kataoka K, Watanabe S, Tanigami A and Sugano S: CREB-H: A novel mammalian transcription factor belonging to the CREB/ATF family and functioning via the box-B element with a liver-specific expression. Nucleic Acids Res 29: 2154-2162, 2001.

14. DenBoer LM, Hardy-Smith PW, Hogan MR, Cockram GP, Audas TE and Lu R: Luman is capable of binding and activating transcription from the unfolded protein response element. Biochem Biophys Res Commun 331: 113-119, 2005.

15. Liang G, Audas TE, Li Y, Cockram GP, Dean JD, Martyn AC, Kokame $\mathrm{K}$ and Lu R: Luman/CREB3 induces transcription of the endoplasmic reticulum (ER) stress response protein Herp through an ER stress response element. Mol Cell Biol 26: 7999-8010, 2006.

16. Kondo S, Murakami T, Tatsumi K, Ogata M, Kanemoto S, Otori K, Iseki K, Wanaka A and Imaizumi K: OASIS, a CREB/ATF-family member, modulates UPR signalling in astrocytes. Nat Cell Biol 7: 186-194, 2005.

17. Kondo S, Saito A, Hino S, Murakami T, Ogata M, Kanemoto S, Nara S, Yamashita A, Yoshinaga K, Hara $\mathrm{H}$ and Imaizumi $\mathrm{K}$ : BBF2H7, a novel transmembrane bZIP transcription factor, is a new type of endoplasmic reticulum stress transducer. Mol Cell Biol 27: 1716-1729, 2007.

18. Nagamori I, Yabuta N, Fujii T, Tanaka H, Yomogida K, Nishimune Y and Nojima H: Tisp40, a spermatid specific bZip transcription factor, functions by binding to the unfolded protein response element via the Rip pathway. Genes Cells 10: 575-594, 2005.

19. Stirling J and O'Hare P: CREB4, a transmembrane bZip transcription factor and potential new substrate for regulation and cleavage by S1P. Mol Biol Cell 17: 413-426, 2006. 
20. Luebke-Wheeler J, Zhang K, Battle M, Si-Tayeb K, Garrison W, Chhinder S, Li J, Kaufman RJ and Duncan SA: Hepatocyte nuclear factor 4alpha is implicated in endoplasmic reticulum stress-induced acute phase response by regulating expression of cyclic adenosine monophosphate responsive element binding protein H. Hepatology 48: 1242-1250, 2008.

21. Bailey D, Barreca C and O'Hare P: Trafficking of the bZIP transmembrane transcription factor CREB-H into alternate pathways of ERAD and stress-regulated intramembrane proteolysis. Traffic 8: 1796-1814, 2007.

22. Zhang K, Shen X, Wu J, Sakaki K, Saunders T, Rutkowski DT, Back SH and Kaufman RJ: Endoplasmic reticulum stress activates cleavage of CREBH to induce a systemic inflammatory response. Cell 124: 587-599, 2006

23. Bailey D and O'Hare P: Transmembrane bZIP transcription factors in ER stress signaling and the unfolded protein response. Antioxid Redox Signal 9: 2305-2321, 2007.

24. Asada R, Kanemoto S, Kondo S, Saito A and Imaizumi K: The signalling from endoplasmic reticulum-resident bZIP transcription factors involved in diverse cellular physiology. J Biochem 149: 507-518, 2011.

25. Chan CP, Mak TY, Chin KT, Ng IO and Jin DY: N-linked glycosylation is required for optimal proteolytic activation of membrane-bound transcription factor CREB-H. J Cell Sci 123 1438-1448, 2010.

26. Barbosa S, Fasanella G, Carreira S, Llarena M, Fox R, Barreca C, Andrew D and O'Hare P: An orchestrated program regulating secretory pathway genes and cargos by the transmembrane transcription factor CREB-H. Traffic 14: 382-398, 2013.

27. Vecchi C, Montosi G, Zhang K, Lamberti I, Duncan SA, Kaufman RJ and Pietrangelo A: ER stress controls iron metabolism through induction of hepcidin. Science 325: 877-880, 2009.

28. Llarena M, Bailey D, Curtis $\mathrm{H}$ and O'Hare P: Different mechanisms of recognition and ER retention by transmembrane transcription factors CREB-H and ATF6. Traffic 11: 48-69, 2010

29. Vecchi C, Montosi G, Garuti C, Corradini E, Sabelli M, Canali S and Pietrangelo A: Gluconeogenic signals regulate iron homeostasis via hepcidin in mice. Gastroenterology 146: 1060-1069, 2014.

30. Lee MW, Chanda D, Yang J, Oh H, Kim SS, Yoon YS, Hong S, Park KG, Lee IK, Choi CS, et al: Regulation of hepatic gluconeogenesis by an ER-bound transcription factor, CREBH. Cell Metab 11: 331-339, 2010.

31. Chanda D, Kim DK, Li T, Kim YH, Koo SH, Lee CH, Chiang JY and Choi HS: Cannabinoid receptor type 1 (CB1R) signaling regulates hepatic gluconeogenesis via induction of endoplasmic reticulum-bound transcription factor cAMP-responsive element-binding protein $\mathrm{H}(\mathrm{CREBH})$ in primary hepatocytes. J Biol Chem 286: 27971-27979, 2011.

32. Chanda D, Kim YH, Kim DK, Lee MW, Lee SY, Park TS, Koo SH, Lee $\mathrm{CH}$ and Choi HS: Activation of cannabinoid receptor type 1 (Cb1r) disrupts hepatic insulin receptor signaling via cyclic AMP-response element-binding protein $\mathrm{H}$ (Crebh)-mediated induction of Lipin1 gene. J Biol Chem 287: 38041-38049, 2012.

33. Zhang, C, Wang G, Zheng Z, Maddipati KR, Zhang X, Dyson G, Williams P, Duncan SA, Kaufman RJ and Zhang K: Endoplasmic reticulum-tethered transcription factor cAMP responsive element-binding protein, hepatocyte specific, regulates hepatic lipogenesis, fatty acid oxidation, and lipolysis upon metabolic stress in mice. Hepatology 55: 1070-1082, 2012.

34. Lee JH, Giannikopoulos P, Duncan SA, Wang J, Johansen CT, Brown JD, Plutzky J, Hegele RA, Glimcher LH and Lee AH The transcription factor cyclic AMP-responsive element-binding protein $\mathrm{H}$ regulates triglyceride metabolism. Nat Med 17: 812-815, 2011.

35. Misra J, Chanda D, Kim DK, Cho SR, Koo SH, Lee CH, Back SH and Choi HS: Orphan nuclear receptor Err $\gamma$ induces C-reactive protein gene expression through induction of ER-bound Bzip transmembrane transcription factor CREBH. PLoS One 9: e86342, 2014.

36. Xu X, Park JG, So JS, Hur KY and Lee AH: Transcriptional regulation of apolipoprotein A-IV by the transcription factor CREBH. J Lipid Res 55: 850-859, 2014

37. Shin DY, Chung J, Joe Y, Pae HO, Chang KC, Cho GJ, Ryter SW and Chung HT: Pretreatment with CO-releasing molecules suppresses hepcidin expression during inflammation and endoplasmic reticulum stress through inhibition of the STAT3 and CREBH pathways. Blood 119: 2523-2532, 2012.
38. Chanda D, Kim YH, Li T, Misra J, Kim DK, Kim JR, Kwon J, Jeong WI, Ahn SH, Park TS, et al: Hepatic cannabinoid receptor type 1 mediates alcohol-induced regulation of bile acid enzyme genes expression via CREBH. PLoS One 8: e68845, 2013.

39. Kim DK, Ryu D, Koh M, Lee MW, Lim D, Kim MJ, Kim YH, Cho WJ, Lee CH, Park TS, et al: Orphan nuclear receptor estrogen-related receptor $\gamma(\mathrm{ERR} \gamma)$ is key regulator of hepatic gluconeogenesis. J Biol Chem 287: 21628-21639, 2012.

40. Kim DK, Kim JR, Koh M, Kim YD, Lee JM, Chanda D, Park SB, Min JJ, Lee CH, Park TS, et al: Estrogen-related receptor $\gamma$ $(E R R \gamma)$ is a novel transcriptional regulator of phosphatidic acid phosphatase, LIPIN1, and inhibits hepatic insulin signaling. J Biol Chem 286: 38035-38042, 2011.

41. Kim DK, Kim YH, Jang HH, Park J, Kim JR, Koh M, Jeong WI, Koo SH, Park TS, Yun $\mathrm{CH}$, et al: Estrogen-related receptor $\gamma$ controls hepatic CB1 receptor-mediated CYP2E1 expression and oxidative liver injury by alcohol. Gut 62: 1044-1054, 2013.

42. Xie YB, Park JH, Kim DK, Hwang JH, Oh S, Park SB, Shong M, Lee IK and Choi HS: Transcriptional corepressor SMILE recruits SIRT1 to inhibit nuclear receptor estrogen receptor-related receptor gamma transactivation. J Biol Chem 284, 28762-28774, 2009.

43. Xie YB, Nedumaran B and Choi HS: Molecular characterization of SMILE as a novel corepressor of nuclear receptors. Nucleic Acids Res 37: 4100-4115, 2009

44. Lu R and Misra V: Zhangfei: A second cellular protein interacts with herpes simplex virus accessory factor HCF in a manner similar to Luman and VP16. Nucleic Acids Res 28: 2446-2454, 2000.

45. Xie YB, Lee OH, Nedumaran B, Seong HA, Lee KM, Ha H, Lee IK, Yun Y and Choi HS: SMILE, a new orphan nuclear receptor SHP-interacting protein, regulates SHP-repressed estrogen receptor transactivation. Biochem J 416: 463-473, 2008.

46. Misra J, Chanda D, Kim DK, Li T, Koo SH, Back SH, Chiang JY and Choi HS: Curcumin differentially regulates endoplasmic reticulum stress through transcriptional corepressor SMILE (small heterodimer partner-interacting leucine zipper protein)-mediated inhibition of CREBH (cAMP responsive element-binding protein H). J Biol Chem 286: 41972-41984, 2011.

47. Danno H, Ishii KA, Nakagawa Y, Mikami M, Yamamoto T, Yabe S, Furusawa M, Kumadaki S, Watanabe K, Shimizu H, et al: The liver-enriched transcription factor CREBH is nutritionally regulated and activated by fatty acids and PPARalpha. Biochem Biophys Res Commun 391: 1222-1227, 2010.

48. Gentile CL, Wang D, Pfaffenbach KT, Cox R, Wei Y and Pagliasotti MJ: Fatty acids regulate CREBh via transcriptional mechanisms that are dependent on proteasome activity and insulin. Mol Cell Biochem 344: 99-107, 2010.

49. Vegiopoulos A and Herzig S: Glucocorticoids, metabolism and metabolic diseases. Mol Cell Endocrinol 275: 43-61, 2007.

50. Wong SW, Kwon MJ, Choi AM, Kim HP, Nakahira K and Hwang DH: Fatty acids modulate Toll-like receptor 4 activation through regulation of receptor dimerization and recruitment into lipid rafts in a reactive oxygen species-dependent manner. J Biol Chem 284: 27384-27392, 2009.

51. Schaeffler A, Gross P, Buettner R, Bollheimer C, Buechler C, Neumeier M, Kopp A, Schoelmerich J and Falk W: Fatty acid-induced induction of Toll-like receptor-4/nuclear factor-kappaB pathway in adipocytes links nutritional signalling with innate immunity. Immunology 126: 233-245, 2009.

52. Miura $\mathrm{K}$ and Ohnishi $\mathrm{H}$ : Role of gut microbiota and Toll-like receptors in nonalcoholic fatty liver disease. World J Gastroenterol 20: 7381-7391, 2014

53. Pineda Torra I, Jamshidi Y, Flavell DM, Fruchart JC and Staels B: Characterization of the human PPARalpha promoter: Identification of a functional nuclear receptor response element. Mol Endocrinol 16: 1013-1028, 2002.

54. Hwang-Verslues WW and Sladek FM: HNF4 $\alpha$-role in drug metabolism and potential drug target? Curr Opin Pharmacol 10 698-705, 2010.

55. Goldberg IJ, Scheraldi CA, Yacoub LK, Saxena U and Bisgaier CL: Lipoprotein ApoC-II activation of lipoprotein lipase. Modulation by apolipoprotein A-IV. J Biol Chem 265: 4266-4272, 1990.

56. Jong MC, Hofker MH and Havekes LM: Role of ApoCs in lipoprotein metabolism: Functional differences between ApoC1, ApoC2, and ApoC3. Arterioscler Thromb Vasc Biol 19: 472-484, 1999. 
57. Marçais C, Verges B, Charrière S, Pruneta V, Merlin M, Billon S, Perrot L, Drai J, Sassolas A, Pennacchio LA, et al: Apoa5 Q139X truncation predisposes to late-onset hyperchylomicronemia due to lipoprotein lipase impairment. J Clin Invest 115: 2862-2869, 2005

58. Merkel M, Eckel RH and Goldberg IJ: Lipoprotein lipase: Genetics, lipid uptake, and regulation. J Lipid Res 43: 1997-2006, 2002.

59. Pennacchio LA, Olivier M, Hubacek JA, Cohen JC, Cox DR Fruchart JC, Krauss RM and Rubin EM: An apolipoprotein influencing triglycerides in humans and mice revealed by comparative sequencing. Science 294: 169-173, 2001.

60. Nishimura T, Nakatake Y, Konishi M and Itoh N: Identification of a novel FGF, FGF-21, preferentially expressed in the liver. Biochim Biophys Acta 1492: 203-206, 2000.

61. Dushay J, Chui PC, Gopalakrishnan GS, Varela-Rey M,Crawley M, Fisher FM, Badman MK, Martinez-Chantar ML and Maratos-Flier $\mathrm{E}$ : Increased fibroblast growth factor 21 in obesity and nonalcoholic fatty liver disease. Gastroenterology 139: 456-463, 2010.

62. Chavez AO, Molina-Carrion M, Abdul-Ghani MA, Folli F, Defronzo RA and Tripathy D: Circulating fibroblast growth factor-21 is elevated in impaired glucose tolerance and type 2 diabetes and correlates with muscle and hepatic insulin resistance. Diabetes Care 32: 1542-1546, 2009.

63. Li H, Dong K, Fang Q, Hou X, Zhou M, Bao Y, Xiang K, Xu A and Jia W: High serum level of fibroblast growth factor 21 is an independent predictor of non-alcoholic fatty liver disease: A 3-year prospective study in China. J Hepatol 58: 557-563, 2013.

64. Matsusue K, Kusakabe T, Noguchi T, Takiguchi S, Suzuki T, Yamano S and Gonzalez FJ: Hepatic steatosis in leptin-deficient mice is promoted by the PPARgamma target gene Fsp27. Cell Metab 7: 302-311, 2008.

65. Puri V, Konda S, Ranjit S, Aouadi M, Chawla A, Chouinard M, Chakladar A and Czech MP: Fat-specific protein 27, a nove lipid droplet protein that enhances triglyceride storage. J Biol Chem 282: 34213-34218, 2007

66. Jambunathan S, Yin J, Khan W, Tamori Y and Puri V: FSP27 promotes lipid droplet clustering and then fusion to regulate triglyceride accumulation. PLoS One 6: e28614, 2011.

67. Reue K: The lipin family: Mutations and metabolism. Curr Opin Lipidol 20: 165-170, 2009.

68. Song KH, Park AY, Kim JE and Ma JY: Identification and characterization of cyclic AMP response element-binding protein $\mathrm{H}$ response element in the human apolipoprotein $\mathrm{A} 5$ gene promoter. BioMed Res Int 2013: 892491,2013.

69. Kim H, Mendez R, Zheng Z, Chang L, Cai J, Zhang R and Zhang K: Liver-enriched transcription factor CREBH interacts with peroxisome proliferator-activated receptor $\alpha$ to regulate metabolic hormone FGF21. Endocrinology 155: 769-782, 2014.

70. Xu X, Park JG, So JS and Lee AH: Transcriptional activation of Fsp27 by the liver-enriched transcription factor CREBH promotes lipid droplet growth and hepatic steatosis. Hepatology 61 : 857-869, 2015

71. Hall RK and Granner DK: Insulin regulates expression of metabolic genes through divergent signaling pathways. J Basic Clin Physiol Pharmacol 10: 119-133, 1999.

72. Hanson RW and Reshef L: Regulation of phosphoenolpyruvate carboxykinase (GTP) gene expression. Annu Rev Biochem 66 : 581-611, 1997.

73. Facchini FS, Hua NW and Stoohs RA: Effect of iron depletion in carbohydrate-intolerant patients with clinical evidence of nonalcoholic fatty liver disease. Gastroenterology 122: 931-939, 2002.

74. Fernández-Real JM, Peñarroja G, Castro A, García-Bragado F, Hernández-Aguado I and Ricart W: Blood letting in high-ferritin type 2 diabetes: Effects on insulin sensitivity and beta-cell function. Diabetes 51: 1000-1004, 2002.

75. Valenti L, Moscatiello S, Vanni E, Fracanzani AL, Bugianesi E, Fargion S and Marchesini G: Venesection for non-alcoholic fatty liver disease unresponsive to lifestyle counselling--a propensity score-adjusted observational study. QJM 104: 141-149, 2011.

76. Jeong WI, Osei-Hyiaman D, Park O, Liu J, Bátkai S, Mukhopadhyay P, Horiguchi N, Harvey-White J, Marsicano G, Lutz B, et al: Paracrine activation of hepatic CB1 receptors by stellate cell-derived endocannabinoids mediates alcoholic fatty liver. Cell Metab 7: 227-235, 2008.

77. Min AK, Jeong JY, Go Y, Choi YK, Kim YD, Lee IK and Park KG: cAMP response element binding protein $\mathrm{H}$ mediates fenofibrate-induced suppression of hepatic lipogenesis. Diabetologia 56: 412-422, 2013.
78. Gabay $\mathrm{C}$ and Kushner I: Acute-phase proteins and other systemic responses to inflammation. N Engl J Med 340: 448-454, 1999.

79. Medzhitov R and Janeway CR Jr: Decoding the patterns of self and nonself by the innate immune system. Science 296: 298-300, 2002.

80. Yoo JY and Desiderio S: Innate and acquired immunity intersect in a global view of the acute-phase response. Proc Natl Acad Sci USA 100: 1157-1162, 2003

81. Kaplan J, Ward DM and De Domenico I: The molecular basis of iron overload disorders and iron-linked anemias. Int J Hematol 93: 14-20, 2011.

82. Kawano Y and Cohen DE: Mechanisms of hepatic triglyceride accumulation in non-alcoholic fatty liver disease. J Gastroenterol 48: 434-441, 2013.

83. Musso G, Gambino R and Cassader M: Recent insights into hepatic lipid metabolism in non-alcoholic fatty liver disease (NAFLD). Prog Lipid Res 48: 1-26, 2009.

84. Donnelly KL, Smith CI, Schwarzenberg SJ, Jessurun J, Boldt MD and Parks EJ: Sources of fatty acids stored in liver and secreted via lipoproteins in patients with nonalcoholic fatty liver disease. J Clin Invest 115: 1343-1351, 2005.

85. Postic C and Girard J: Contribution of de novo fatty acid synthesis to hepatic steatosis and insulin resistance: Lessons from genetically engineered mice. J Clin Invest 118: 829-838, 2008.

86. Tiwari S and Siddiqi SA: Intracellular trafficking and secretion of VLDL. Arterioscler Thromb Vasc Biol 32: 1079-1086, 2012.

87. Jornayvaz FR and Shulman GI: Diacylglycerol activation of protein kinase $C \varepsilon$ and hepatic insulin resistance. Cell Metab 15: 574-584, 2012.

88. Neuschwander-Tetri BA: Hepatic lipotoxicity and the pathogenesis of nonalcoholic steatohepatitis: The central role of nontriglyceride fatty acid metabolites. Hepatology 52: 774-788, 2010.

89. Zámbó V, Simon-Szabó L, Szelényi P, Kereszturi E, Bánhegyi G and Csala M: Lipotoxicity in the liver. World J Hepatol 5: 550-557, 2013.

90. Gregor MF, Yang L, Fabbrini E, Mohammed BS, Eagon JC, Hotamisligil GS and Klein S: Endoplasmic reticulum stress is reduced in tissues of obese subjects after weight loss. Diabetes 58: 693-700, 2009.

91. Puri P, Mirshahi F, Cheung O, Natarajan R, Maher JW, Kellum JM and Sanyal AJ: Activation and dysregulation of the unfolded protein response in nonalcoholic fatty liver disease. Gastroenterology 134: 568-576, 2008.

92. Sharma NK, Das SK, Mondal AK, Hackney OG, Chu WS, Kern PA, Rasouli N, Spencer HJ, Yao-Borengasser A and Elbein SC: Endoplasmic reticulum stress markers are associated with obesity in nondiabetic subjects. J Clin Endocrinol Metab 93: 4532-4541, 2008

93. Wang D, Wei Y and Pagliassotti MJ: Saturated fatty acids promote endoplasmic reticulum stress and liver injury in rats with hepatic steatosis. Endocrinology 147: 943-951, 2006.

94. Brunt EM, Janney CG, Di Bisceglie AM, Neuschwander-Tetri BA and Bacon BR: Nonalcoholic steatohepatitis: A proposal for grading and staging the histological lesions. Am J Gastroenterol 94: 2467-2474, 1999.

95. Kleiner DE, Brunt EM, Van Natta M, Behling C, Contos MJ, Cummings OW, Ferrell LD, Liu YC, Torbenson MS Unalp-Arida A, et al; Nonalcoholic Steatohepatitis Clinical Research Network: Design and validation of a histological scoring system for nonalcoholic fatty liver disease. Hepatology 41: 1313-1321, 2005

96. Sakaguchi S, Takahashi S, Sasaki T, Kumagai T and Nagata K: Progression of alcoholic and non-alcoholic steatohepatitis: Common metabolic aspects of innate immune system and oxidative stress. Drug Metab Pharmacokinet 26: 30-46, 2011.

97. Shimomura I, Bashmakov Y and Horton JD: Increased levels of nuclear SREBP-1c associated with fatty livers in two mouse models of diabetes mellitus. J Biol Chem 274: 30028-30032, 1999.

98. de Luca C and Olefsky JM: Inflammation and insulin resistance. FEBS Lett 582: 97-105, 2008

99. Polyzos SA, Kountouras J and Zavos C: Nonalcoholic fatty liver disease: The pathogenetic roles of insulin resistance and adipocytokines. Curr Mol Med 9: 299-314, 2009. 